\title{
“GECKOFLUIDICS": A NEW CONCEPT IN REVERSIBLE BONDING OF MICROFLUIDIC CHANNELS
}

\author{
A. Wasay*, and D. Sameoto
}

Department of Mechanical Engineering, University of Alberta, Edmonton, Alberta, Canada

\begin{abstract}
Reversibly sealed PDMS microfluidics are attractive because they solve the bonding challenges that are so difficult with rigid thermoplastics. Unfortunately, reversible bonding with PDMS is also problematic, because the channels cannot hold high pressures. In this work, we demonstrate a significant advancement through the application of gecko-inspired adhesives to strongly enhance polymer microfluidic reversible bonding and apply it with thermoplastic elastomers in a simple thermocompression molding process. This technique is applied to thermoplastic elastomers which have recently demonstrated great promise as a replacement material for PDMS in many microfluidic applications.
\end{abstract}

\section{INTRODUCTION}

Reversible microfluidic bonding with elastomer materials is easy, but typically very weak [1]. A strong, reversible bond may be considered a contradiction in terms, but is in fact achievable through the use of special contact geometries as employed in the field of gecko-inspired adhesives. Using a recently developed manufacturing method for thermoplastic elastomer dry adhesives [2], we demonstrate how these materials can be employed to turn any nearly flat surface into a microfluidic system with minimal effort.

For a typical reversible bonded channel in an elastomer based microfluidic system, a channel wall simply meets the bonded surface with a flat punch type contact tip at an angle of approximately $90^{\circ}$. This type of geometry is extremely vulnerable to stress concentrations at the contact edge which will cause much earlier adhesive failure than the theoretical strength possible via van der Waals forces [3]. An overhanging cap however strongly reduces the stress concentrations at the edge and can permit reversible adhesion of fibers approaching $1 \mathrm{MPa}$ in axial loading. Through the design of a single "fiber" that is continuous around the perimeter of the desired channel shape, we can define a selfsealing gasket which will contain fluid. This gasket can be surrounded by further fibers to enhance the total adhesion and help make the rest of the surface more tolerant to defects and surface roughness. This mechanism strongly improves the total performance of the reversible bond.

\section{FABRICATION}

The fabrication steps are similar to those reported earlier for the fabrication of a master mold [4], and the thermocompression molding of a thermoplastic elastomer [2]. With the choice of an appropriate thermoplastic elastomer that will flow well at elevated temperatures, even highly interlocked features can be molded without large amounts of trapped gas. The "geckofluidics" concept has thus far been demonstrated with two types of styreneethylene-butylene-styrene (SEBS) elastomers, Kraton G1657 and G1645, but other materials could be used in future. Thermocompression molding time typically requires no more than a couple minutes total to produce the channels once the silicone mold is prepared. The molding is done using a Branson ultrasonic welder without application of ultrasonic energy, which can apply between $15-448 \mathrm{lbs}$ force on a pre-heated hotplate containing the silicone molds and elastomer pucks.

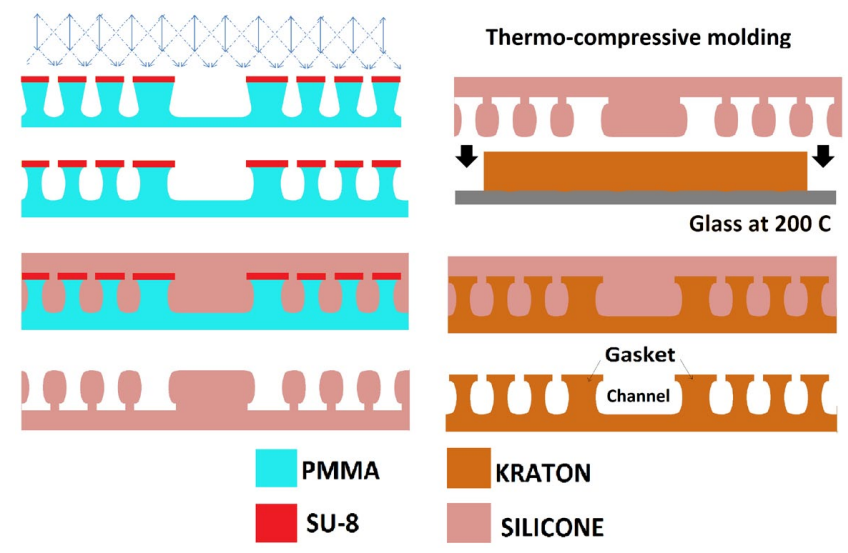

Figure 1: An SU-8 coated acrylic substrate is exposed with a semicollimated $254 \mathrm{~nm}$ light and developed in solvent to produce a master mold with individual adhesive fibers and continuous features that serve as channel walls. Channels can range in depth from several to over $100 \mu \mathrm{m}$. A silicone negative (TC-5030 from $B J B$ Enterprises) is cast from this master then demolded. The silicone negative is used for thermo-compression molding of Kraton G1657 or G1645 elastomer. Pellets of Kraton resin are first melted against a glass microscope slide on a hot plate at 200 ${ }^{\circ} \mathrm{C}$, and then the silicone mold is placed on top. Once the mold and pellets are preheated, the silicone mold is pressed down for 30 seconds at variable force depending on the device size and desired thickness. After cooling and demolding, the elastomer geckofluidics can be peeled off the glass slide and are reversibly bonded to any relatively smooth surface.

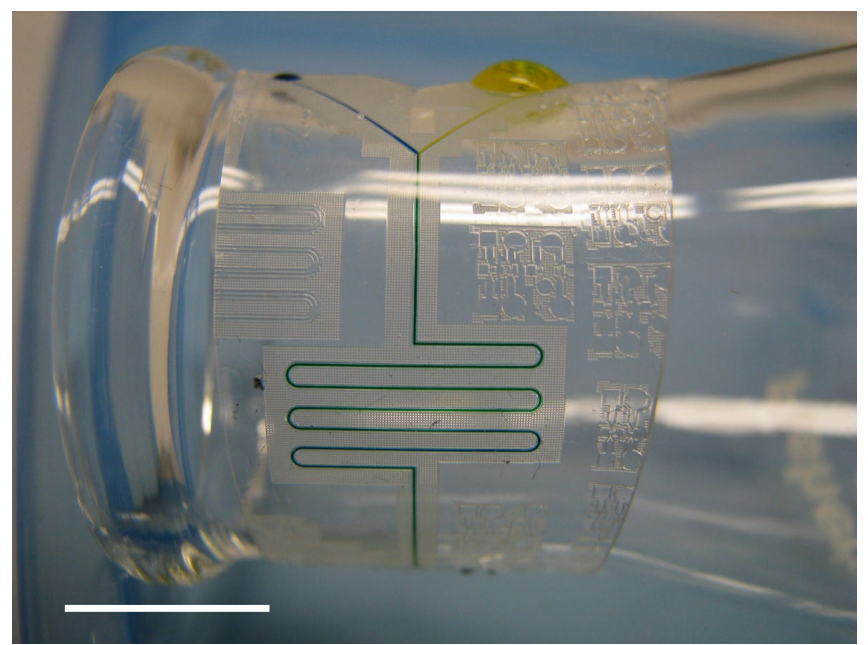

Figure 2: A micro-mixer, showing the mixing of two food dyes to produce a homogeneous blend. The whole design is attached reversibly and strongly to the neck of a clean Erlenmeyer flask demonstrating the versatility and flexibility of the technique. For example, in this case, gravity driven flow can assist in filling the channels of a micromixer while keeping large drops contained on the top surface. The scale bar is $1 \mathrm{~cm}$. 


\section{RESULTS}

Once the microfluidics are demolded from the mold, they can have holes opened in SEBS reservoirs with a punch (Fig. 2-4), or be simply bonded like a sticker over through holes that are already drilled or cut into prepared substrates. For an initial proof of concept design, a polystyrene petri dish lid was sputtered with 20 $\mathrm{nm}$ of gold through a stencil to form electrodes to match the reservoirs of a capillary electrophoresis channel adapted from previous work [5] (Fig. 3). Wires were soldered to the gold electrodes with Field's metal. Future trials will be completed to compare with other recently reported work on SEBS electrokinetic applications [6]. Well-designed adhesive pillars permit extreme reversible peel strength of geckofluidics (Fig. 4). A rigid backing layer may be added to transfer load from the gaskets (Fig. 5) to the array of pillars and improve sealing against higher pressure.

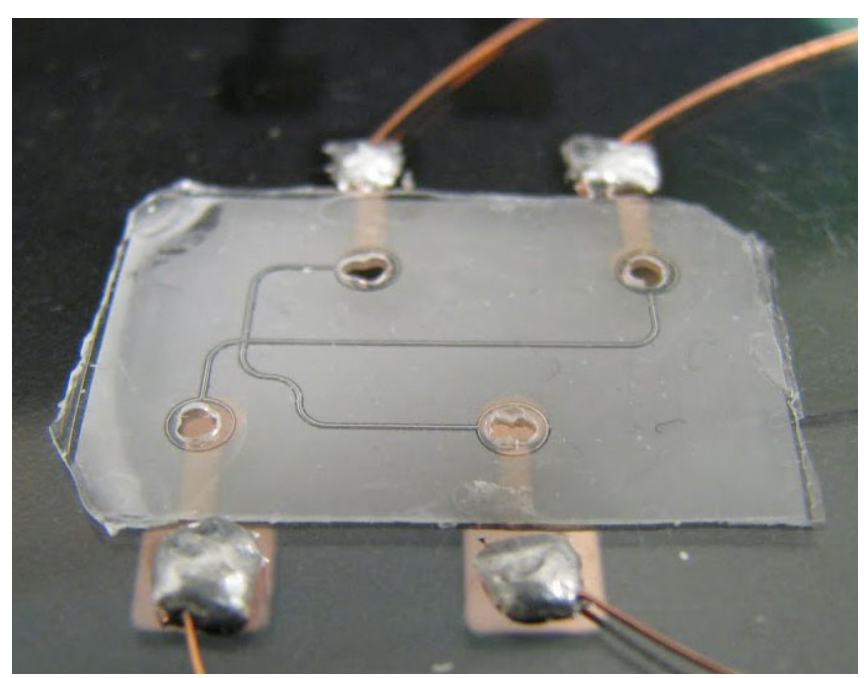

Figure 3: A capillary electrophoresis design adapted from other work [5] showing reversibly bonded channels on top of electrodes.

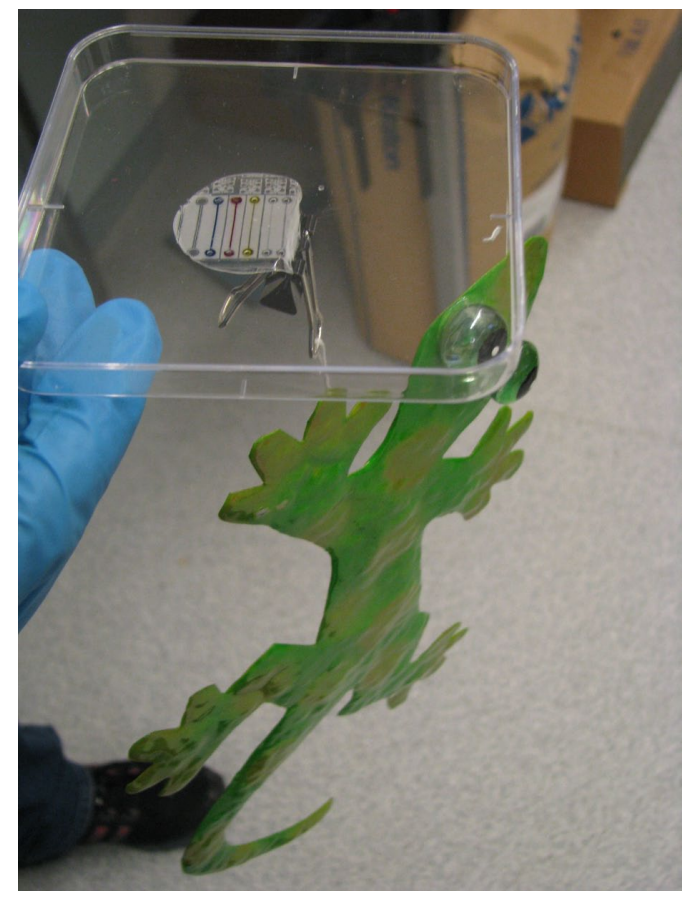

Figure 4: A demonstrative view of geckofluidics with dyed channels under a peeling load of 75 grams

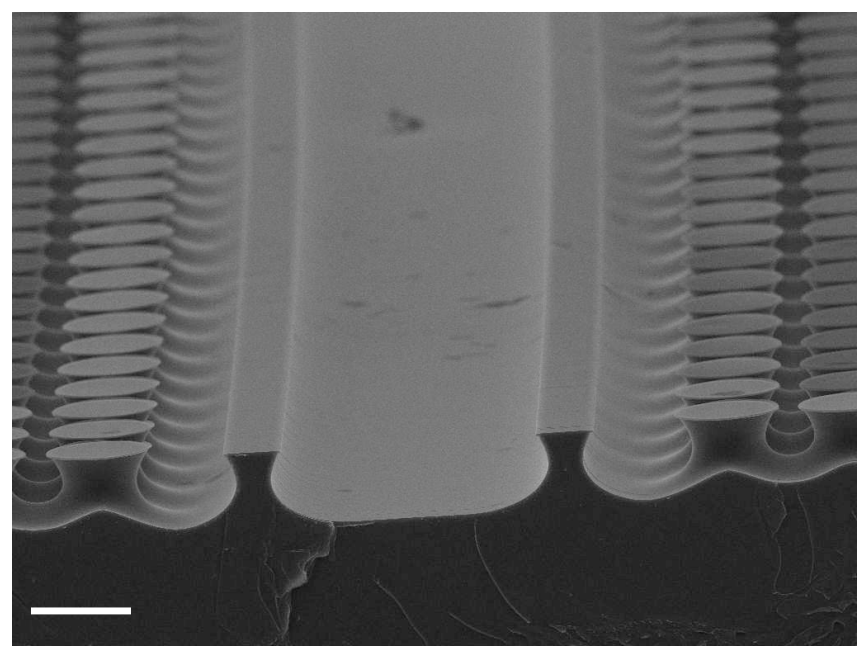

Figure 5: Scanning electron microscope image of SEBS pillars and gaskets for geckofluidics. Some debris is seen from the cutting process, but otherwise channels and cap surfaces are smooth for quality bonding and good imaging. Scale bar represents $100 \mu \mathrm{m}$.

\section{CONCLUSIONS}

We have demonstrated a novel and extremely effective fabrication technology for developing reversible bonded microfluidics with strong resistance to leakage and easy integration with electrodes. Because it can be bonded to any relatively smooth surface with minimal effort, it is a solution that could be integrated with CMOS or silicon MEMS with few modifications.

\section{ACKNOWLEDGEMENTS}

We would like to thank NSERC for financial support and $\mathrm{CMC}$ microsystems for test support through access to their microsystems integration platform (MIP).

\section{REFERENCES}

[1] J.C. McDonald, M.L. Chabinyc, S. Metallo, J. R. Anderson, A. D. Stroock, and G. M. Whitesides, "Prototyping of microfluidic devices in poly(dimethylsiloxane) using solidobject printing" Anal. Chem., 74, 1537, (2002)

[2] W. Khaled, and D. Sameoto, "Manufacture of mushroom shaped dry adhesives by thermo-compression molding of thermoplastic elastomers", Proc. of the Adhesion Society, February 23-26, (2014). San Diego CA.

[3] G. Carbone, E. Pierro, and S. N. Gorb, "Origin of the superior adhesive performance of mushroom-shaped microstructured surfaces." Soft Matter, 7, 5545 (2011)

[4] D. Sameoto and C. Menon, "Deep UV patterning of acrylic masters for molding biomimetic dry adhesives", J. Micromech. Microeng., 20, 115037, (2010)

[5] Behnam, M.; Kaigala, G.V.; Khorasani, M.; Martel, S.; Elliott, D.G.; Backhouse, C.J., "Integrated circuit-based instrumentation for microchip capillary electrophoresis," Nanobiotechnology, IET, 4, 91, (2010)

[6] M. D. Borysiak, E. Yuferova, and J. D. Posner, "Simple, Low-Cost Styrene-Ethylene/Butylene-Styrene Microdevices for Electrokinetic Applications", Anal. Chem 85, 11700 (2013)

\section{CONTACT}

D. Sameoto, tel: +1-780-248-2052; sameoto@ualberta.ca 